\title{
Cost-effectiveness of adding daratumumab or bortezomib to lenalidomide plus dexamethasone for newly diagnosed multiple myeloma
}

\author{
Nihal Narsipur, PharmD; Sabrina Bulla, BA; Connie Yoo, BA; Brenda Do, BS; Kyle Tran, BS; Dian Gu, PhD; \\ Lixian Zhong, PhD; and Leslie Wilson, PhD
}

\section{What is already known about this subject}

- Daratumumab added to lenalidomide and dexamethasone significantly improves progression-free survival for newly diagnosed multiple myeloma patients compared with lenalidomide and dexamethasone alone.

- The National Comprehensive Cancer Network recommends triple therapy regimens of bortezomib added to lenalidomide and dexamethasone, triple therapy of daratumumab, lenalidomide, and dexamethasone, or dual therapy lenalidomide and dexamethasone as preferred treatment options for the treatment of newly diagnosed multiple myeloma patients ineligible for autologous stem cell transplantation.

- From previous cost-effectiveness studies, the triple therapy regimen of daratumumab added to lenalidomide and dexamethasone has not been shown to be cost-effective compared with lenalidomide and dexamethasone in patients with relapsed, refractory multiple myeloma.

\section{What this study adds}

- This study evaluates the costeffectiveness of both daratumumab and bortezomib in combination with lenalidomide and dexamethasone for the treatment of newly diagnosed multiple myeloma patients ineligible for autologous stem cell transplantation.

- This study compares the 3 treatment regimens recommended by the National Comprehensive Cancer Network guidelines for newly diagnosed multiple myeloma patients ineligible for stem cell transplantation.

- The direct comparison between multiple regimens provides essential guidance to payers, clinicians, and formulary decision makers, regarding which regimen is most cost-effective.

\section{ABSTRACT}

BACKGROUND: Multiple myeloma survival rates are steadily increasing due to availability of new drug classes used in combination with corticosteroids and chemotherapy. The latest treatments are daratumumab or bortezomib in combination therapy with lenalidomide and dexamethasone (Rd). Daratumumab, a CD38-targeted, human IgGlk monoclonal antibody, and bortezomib, a proteasome inhibitor, are both approved as regimens for transplant-ineligible relapsed/ refractory multiple myeloma (RRMM). There have been cost-effectiveness analyses for daratumumab and bortezomib use in

\author{
Author affiliations \\ Nihal Narsipur, PharmD, UCSF-Actelion \\ Clinical Research and Medical \\ Communications Fellow, University of \\ California, San Francisco. Sabrina Bulla, \\ BA; Connie Yoo, BA; Brenda Do, BS; and \\ Kyle Tran, BS, PharmD Candidates 2021, \\ University of California, San Francisco. Dian \\ Gu, PhD, Institute for Health and Aging, and \\ Leslie Wilson, PhD, Department of Clinical \\ Pharmacy, University of California, San \\ Francisco. Lixian Zhong, PhD, College of \\ Pharmacy, Texas A\&M University.
}

AUTHOR CORRESPONDENCE:

Leslie Wilson, 415.990.1012;

leslie.wilson@ucsf.edu

J Manag Care Spec Pharm. 2021;27(12):1691-702

Copyright $\odot 2021$, Academy of Managed Care Pharmacy. All rights reserved.

RRMM, but there are limited data regarding cost-effectiveness for daratumumab or bortezomib use in newly diagnosed multiple myeloma patients who are ineligible for stem cell transplantation.

OBJECTIVE: To compare the cost-effectiveness of 3 separate regimens-(1) daratumumab, 
lenalidomide, and dexamethasone triple therapy (DRd); (2) bortezomib and lenalidomide plus dexamethasone triple therapy (VRd); and (3) lenalidomide plus dexamethasone ( $\mathrm{Rd}$ ) - in patients with multiple myeloma ineligible for autologous stem cell transplant.

METHODS: A 2-state Markov model was developed using a US health system perspective and lifetime time horizon. Transition probabilities were calculated from the latest progression-free survival data reported in two phase 3 randomized controlled trials-MAIA and SWOG S0777-and extrapolated using a Weibull distribution based on the Hoyle Henley method. National data sources were used to obtain costs in 2019 US dollars, discounted by $3 \%$. Health state utilities from available literature were applied to each health state. Utility decrements for adverse events were individualized in each choice branch with utility decrement weighted by the percentage of patients who experienced the adverse event in the MAIA and SWOG S0777 trials. We assumed a treatment would be cost-effective at a willingness to pay (WTP) of $\$ 150,000$ per progression-free qualityadjusted life-year (\$/PFQALY). One-way and probabilistic sensitivity analyses were conducted.

RESULTS: Rd standard therapy had the lowest overall cost at $\$ 329,867$, followed by VRd at $\$ 385,434$ and DRd with the highest overall total cost at $\$ 626,900$. Rd was estimated to result in the least amount (1.24) of PFQALYs, followed by VRd at 1.35 PFQALYs and DRd at 1.52 PFQALYs. With a WTP threshold of $\$ 150,000$ per PFQALY, VRd was not cost-effective compared with Rd standard therapy, with an incremental cost-effectiveness ratio (ICER) of $\$ 530,256$ per PFQALY. DRd was not cost-effective compared with VRd (ICER $=\$ 1,396,318$ per PFQALY), nor as compared with Rd standard therapy $($ ICER $=\$ 1060,832)$. One-way sensitivity analysis showed that our model was sensitive to cost of DRd, VRd, and Rd drugs. Probabilistic sensitivity analysis showed that only at a WTP threshold of $\$ 550,000$ was VRd cost-effective for $40 \%$ of iterations. There were no reasonable WTP thresholds, up to $\$ 800,00$, where DRd became more cost-effective than VRd.

CONCLUSIONS: This study is the first analysis to directly compare the cost-effectiveness of 3 acceptable chemotherapy treatment regimens for patients with multiple myeloma ineligible for autologous stem cell transplant. Neither DRd nor VRd triple therapy were found to be cost-effective vs Rd. Further cost-effectiveness analyses that include overall survival data for daratumumab and bortezomib triple therapies are needed to demonstrate an ICER in QALYs.

Multiple myeloma is a hematologic malignancy that is characterized by the proliferation of malignant plasma cells in the bone marrow and accounts for approximately $13 \%$ of hematologic cancers. ${ }^{1,2}$ In the United States, there were an estimated 32,270 new cases of multiple myeloma and 12,830 deaths related to multiple myeloma in $2020 .^{3}$ Within the past decade, there has been a $35 \%$ decline in mortality, but treatment costs for multiple myeloma have increased by
$26 \% .^{4}$ Despite recent advances in the treatment of multiple myeloma, it remains largely incurable with a 5-year survival rate of only $52.2 \% .^{5}$

Based on the National Comprehensive Cancer Network Multiple Myeloma Guidelines, preferred treatment for multiple myeloma patients is high-dose chemotherapy followed by 2 autologous stem cell transplantations (ASCT). ${ }^{6,7}$ However, more than half of patients with newly diagnosed multiple myeloma (NDMM) are deemed ineligible for ASCT because of common comorbidities and complications of multiple myeloma. ${ }^{8}$ Standard-risk patients with NDMM ineligible for ASCT are generally recommended for treatment initiation of a 2-drug regimen of lenalidomide and dexamethasone $(\mathrm{Rd}){ }^{9}{ }^{9}$

However, a triple therapy with bortezomib (V) or daratumumab (D), combined with the lenalidomide and dexamethasone (Rd) backbone (VRd and DRd, respectively) can now also be considered for patients with NDMM, who are ineligible for ASCT, with category 1 recommendations (grade based on high-level evidence). ${ }^{9}$ In 2010, VRd was shown to be an effective treatment for patients with NDMM compared with $\mathrm{Rd} .{ }^{10}$ In 2018, 2 separate landmark clinical trials demonstrated the effectiveness of daratumumab therapy added to bortezomib, melphalan, and prednisone and daratumumab therapy added to $\mathrm{Rd}$ in patients with NDMM.11-13

Currently, there is no head-to-head clinical trial and no cost-effectiveness analysis across all the newly recommended treatment regimens for patients with NDMM. ${ }^{14}$ Our study goal was to determine if DRd is cost-effective compared with guideline-recommended treatments, VRd and Rd in patients with NDMM ineligible for ASCT, by combining data from the 2 available trials that compare VRd with Rd and DRd with Rd in this sample. ${ }^{9,14}$ This study will help to understand the comparative value of these treatments in order to provide information that can guide treatment decisions by health care providers for this patient population.

\section{Methods}

\section{STUDY POPULATION}

Our Markov model analysis is based on two phase 3 randomized, open-label trials, MAIA and SWOG S0777, both of which contained a study population of patients with NDMM ineligible for ASCT. The MAIA and SWOG S0777 trials had no planned differences in the inclusion and exclusion criteria across studies. However, there are slight differences in demographics between the final study populations in the MAIA (DRd vs Rd comparison) and SWOG S0777 (VRd vs Rd comparison) trials. The MAIA trial had more ISS stage II and 
fewer ISS stage III patients. The median age in the SWOG S077 and MAIA trials was 63 and 73 years, respectively, giving the average age of entry for patients into the Markov model of 68 years. ${ }^{13,15}$

The study population in the MAIA trial was randomized to receive either DRd, the triple therapy arm, or Rd, the standard of care arm. Patients in the DRd arm received 28-day cycles of intravenous daratumumab $16 \mathrm{mg} / \mathrm{kg}$ once weekly for cycles 1 and 2, every 2 weeks for cycles 3 through 6 , and every 4 weeks thereafter; lenalidomide $25 \mathrm{mg}$ for 21 days; and dexamethasone $40 \mathrm{mg}$ for 4 days, in addition to pre-infusion and post-infusion medications..$^{13}$

The study population in the SWOG S077 trial was randomized to receive either 21-day cycles of VRd, the triple therapy arm, or 28-day cycles of Rd, the standard of care arm. Patients in the VRd arm received intravenous bortezomib $1.3 \mathrm{mg} / \mathrm{m}^{2} 4$ times per cycle; oral lenalidomide $25 \mathrm{mg}$ for 14 days; and oral dexamethasone $20 \mathrm{mg}$ for 8 days, in addition to herpes simplex virus prophylaxis and oral aspirin $325 \mathrm{mg}$ to reduce the risk of thromboembolic events. ${ }^{15}$

Both studies had a common comparative arm receiving Rd therapy consisting of oral lenalidomide $25 \mathrm{mg}$ for 21 days and oral dexamethasone $40 \mathrm{mg}$ for 4 days per cycle (Supplementary Table 1, available in online article). However, patients in the Rd arm of the SWOG S077 additionally received $325 \mathrm{mg}$ oral aspirin once a day. ${ }^{13}$

\section{MARKOV MODEL}

We developed a 2-state Markov model with 3 choice branches at the decision node-DRd, VRd, or Rd-using TreeAge Pro 2020 (TreeAge Software). The patient populations, treatment, and dosing regimens included in the model reflect the protocol used in the MAIA and SWOG S077 trials and from the products package inserts. ${ }^{16,17} \mathrm{~A}$ cycle of 28 days was used in the model, since Rd-based treatments are administered in 28-day cycles. The 21-day cycle of the VRd regimen was converted to a 28 -day cycle by multiplying the values going into the model by 1.33 ( 28 days over 21 days) to account for the 7 fewer days in the cycle.

All patients began in the progression-free $(\mathrm{PF})$ health state and either stayed in PF or transitioned to the progressed or dead health state (Supplementary Figure 1, available in online article), ${ }^{4,15,18} \mathrm{~A}$ half-cycle correction was implemented to correct for overestimations. We determined the incremental cost-effectiveness ratio (ICER) of the 3 comparisons using the formula ICER=change in cost/ change in effect. Our main outcome was PF quality-adjusted life-years (PFQALY) saved over the lifetime horizon. Our model took a health system perspective and costs were in US dollars.
Cost-effectiveness was assessed at a willingness-topay (WTP) threshold of $\$ 150,000$, using the World Health Organization's recommendation to set the WTP threshold at 3 times the per capital annual income of a country (approximately $\$ 50,000$ in the United States). ${ }^{19}$ Although this recommendation is for QALYs rather than PFQALYs, there is no recommendation yet for a WTP for PFQALYs. However, estimates of the probability of new cancer drug comparisons remaining significantly positive when moving from PF survival (PFS) outcomes to overall survival (OS) outcomes is over $60 \%$. In addition, when moving from PFS to OS, we expected the survival difference among treatment and control drugs to narrow, whereas cost differences would remain similar. Given these 2 expectations, the WTP level should remain the same for PFS as OS, or even be more generous. Therefore, we used the standard WTP in this study.

We conducted a 1-way sensitivity analysis on key costs and utilities to examine their effects on our model outcomes (Table 1). Probabilistic sensitivity analysis (PSA) using a Monte Carlo simulation with 10,000 iterations was conducted to obtain a cost-effectiveness acceptability curve. We also looked at PF life-years (PFLY) saved over the lifetime horizon (Supplementary Table 3 , available in online article).

\section{TRANSITION PROBABILITIES}

To calculate transition probabilities, the PFS Kaplan-Meier (KM) curves for the treatment and active control arms in the MAIA and SWOG S0777 trials were extracted using a graphical digitizer (Engauge Digitizer, version 12; Mark Mitchell, Baurzhan Muftakhidinov and Tobias Winchen et al). The spreadsheet developed by Martin Hoyle and William Henley was used to estimate individual patient data from the KM curves. $^{20}$ The estimated individual data points were then used to generate the scale and shape parameters for the most suitable model of 4 options: log-logistic, Weibull, lognormal, and logistic. The most suitable model for each PFS curve was chosen based on the lowest Bayesian information criterion and Akaike information criterion.

The modeled PFS curves were adjusted to account for the different trial lengths and rates of censoring in the MAIA and SWOG S0777 trials, by averaging the scale and shape parameters of the common comparator (Figure 1). The adjusted Rd survival curve scale parameter (0.0197) was derived by averaging the original scale parameters from the MAIA and SWOG S077 trials. The adjusted Rd survival curve shape parameter (1.0465) was derived by averaging the original shape parameters from the MAIA and SWOG S0777 trials. ${ }^{13,15}$ 


\section{FIGURE 1 Adjusted and Unadjusted PFS Curves for DRd, VRd, and Rd}

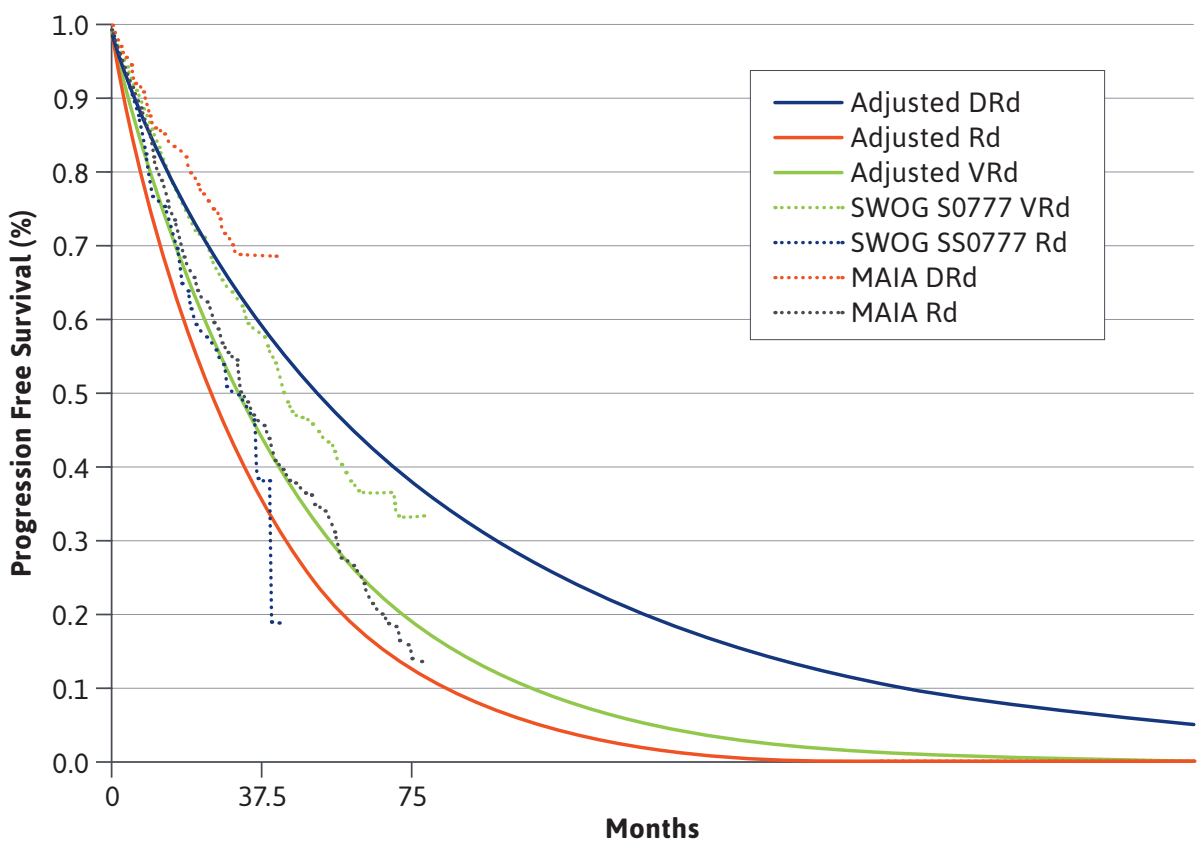

$D R d=$ daratumumab/lenalidomide/dexamethasone; ICER = incremental cost-effectiveness ratio; $P F S=$ progression-free survival; $R d=$ lenalidomide/dexamethasone; $V R d=$ bortezomib/lenalidomide/ dexamethasone.

The adjusted scale and shape parameters of the DRd and VRd survival curves were derived by multiplying the original parameters by a conversion ratio. The conversion ratio was the adjusted Rd parameter divided by the original $\mathrm{Rd}$ parameter from its respective trial (Supplementary Table 2, available in online article). These distributions were then used to extend the PFS curves to lifetime curves and used to calculate transition probabilities for each monthly cycle of the Markov model.

\section{COSTS AND UTILITIES}

All costs were updated to 2019 US dollars using the medical care component of the Consumer Price Index. Average wholesale price (AWP) drug costs in 2019 US dollars were taken from RED BOOK (IBM), with a $16 \%$ discount applied to reflect contract pricing and to be consistent with estimates for Medicare reimbursement. ${ }^{21}$ All costs and health outcomes were discounted $3 \%$ annually. ${ }^{22}$ Drug treatment protocols were taken from MAIA for the DRd regimen and from SWOG S0777 for the VRd regimen. Any weightbased costs were calculated based on the number of vials needed to dose a standard patient with a weight of $70 \mathrm{~kg}$ and body surface area of $1.7 \mathrm{~m}^{2}$. Drug costs per cycle were calculated as the sum of drug therapies, pre-infusion medications, and necessary prophylaxis medications (Table 1).

We used the adverse event (AE) data from the MAIA and SWOG S0777 trials, which reported grade 3 or 4 AEs. ${ }^{13,15}$ The AEs for the Rd arm were derived from AEs in the Rd groups that occurred with a frequency greater than 10\% in the MAIA and SWOG S077 trials. The length of treatment for AEs was derived mainly from previous cost-effectiveness studies with similar side effects and treatment guidelines (Table 1). The main AEs were lymphopenia and sensory neuropathy for VRd and neutropenia for Rd. Utilization and costs for grade 3 or $4 \mathrm{AE}$ management included the use of drugs to treat side effects, laboratory tests to diagnose and monitor the AEs, hospitalization, outpatient visits, and physician fees (Table 1) and were based on treatment guidelines, literature, and expert opinion.

Current Procedural Terminology codes for laboratory tests and outpatient visits were used to identify costs to Medicare from the 2019 University of Pittsburgh Medical Center Lab Fee Schedule and American Medical Association's Resource-Based Relative Value Scale Data Manager and discounted $3 \%$ annually. ${ }^{23}$ Hospitalization visits for all grade 3 or 4 AEs were determined to be high severity. The mean cost of hospitalization and mean length of stay were determined using the Healthcare Utilization and Cost Project (HCUP) and International Classification of Diseases, Tenth Revision, Clinical Modification codes for each AE. ${ }^{24}$ The cost was then adjusted for inflation using the Consumer Price Index. ${ }^{25}$ Hospitalizations were followed by outpatient visits at every 2 weeks.

In addition, we included a one-time progression cost that included supportive care for all the patients that moved to the progression or dead branch. Best supportive care included tests for diagnosis of progression, palliative pain treatment using radiation therapy, and bisphosphonate therapy (Table 1). ${ }^{9,26}$

Health state utilities based on the EuroQol-5 Dimensions Questionnaire were derived from publicly available 


\section{TABLE 1 Model Input Parameters Including Base Case, Ranges for Sensitivity Analysis, and Distributions for Probabilistic Sensitivity Analysis}

\begin{tabular}{|c|c|c|c|c|}
\hline \multirow[b]{2}{*}{ Parameter } & \multirow[b]{2}{*}{ Base case } & \multicolumn{2}{|c|}{ Range $^{a}$} & \multirow[b]{2}{*}{ Source } \\
\hline & & Low value & High value & \\
\hline \multicolumn{5}{|l|}{ Drug costs ( $y$ distribution), $\$$} \\
\hline DRd triple therapy (cycle 1-6) & 62,223 & 46,667 & 77,779 & \multirow{5}{*}{$\begin{array}{l}\text { 23-24, } \\
\text { RED BOOK }\end{array}$} \\
\hline DRd triple therapy (cycle 7 or more) & 22,352 & 16,764 & 27,941 & \\
\hline Rd doublet therapy & 16,191 & 12,143 & 20,239 & \\
\hline VRd triple therapy & 17,243 & 12,932 & 21,554 & \\
\hline VRd triple therapy HSV prophylaxis & 612 & 459 & 765 & \\
\hline \multicolumn{5}{|l|}{ Cost of managing AEs ( $\gamma$ distribution), $\$$} \\
\hline Anemia for DRd & 1,061 & 796 & 1,326 & \multirow{12}{*}{$\begin{array}{c}\text { 16, 19, } \\
\text { HCUP, UPMC Lab Fee } \\
\text { Schedule, AMA RBRVS } \\
\text { Data Manager }\end{array}$} \\
\hline Neutropenia for DRd & 9,248 & 6,936 & 11,560 & \\
\hline Lymphopenia for DRd & 1,388 & 1,041 & 1,735 & \\
\hline Nonhematologic AEs for DRd & 3,066 & 2,299 & 3,832 & \\
\hline Anemia for $\mathrm{Rd}$ & 1,605 & 1,204 & 2,006 & \\
\hline Neutropenia for Rd & 5,206 & 3,905 & 6,508 & \\
\hline Lymphopenia for Rd & 1,591 & 1,193 & 1,989 & \\
\hline Nonhematologic AEs for Rd & 6,324 & 4,743 & 7,905 & \\
\hline Anemia for VRd & 1,169 & 877 & 1,461 & \\
\hline Neutropenia for VRd & 3,514 & 2,635 & 4,393 & \\
\hline Lymphopenia for VRd & 2,114 & 1,586 & 2,643 & \\
\hline Nonhematologic for VRd & 4,207 & 3,155 & 5,259 & \\
\hline Cost of progression ( $y$ distribution), $\$$ & 30,481 & 22,861 & 38,101 & 12,25 \\
\hline Cost of supportive care ( $y$ distribution), $\$$ & 2,199 & 1,650 & 2,749 & 12,25 \\
\hline \multicolumn{5}{|l|}{ 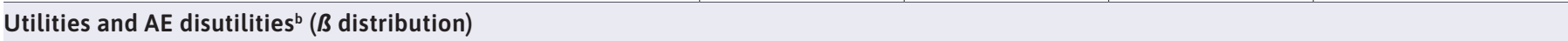 } \\
\hline Baseline alive with no progression for DRd, Rd, or VRd & 0.81 & 0.61 & 1.01 & \multirow{5}{*}{$18,26-31$} \\
\hline Progressed or dead for DRd, Rd, or VRd & 0.701 & 0.53 & 0.88 & \\
\hline Disutility for DRd AEs & 0.12 & 0.09 & 0.15 & \\
\hline Disutility for Rd AEs & 0.07 & 0.05 & 0.09 & \\
\hline Disutility for VRd AEs & 0.09 & 0.07 & 0.11 & \\
\hline
\end{tabular}

${ }^{a} \pm 25 \%$ of baseline was used to calculate low and high values for sensitivity analysis.

${ }^{b}$ Calculated by multiplying the expected disutility for each AE by the proportion of participants who reported grade 3 or 4 AEs and then summing all of the respective AEs for each treatment group.

$A E=$ adverse event; AMA RBRVS=American Medical Association's Resource-Based Relative Value Scale; DRd=daratumumab/lenalidomide/dexamethasone; HCUP = Healthcare Utilization and Cost Project; HSV=herpes simplex virus; $R d=$ lenalidomide/dexamethasone; UPMC=University of Pittsburgh Medical Center; $\mathrm{VRd}=$ bortezomib/lenalidomide/dexamethasone.

literature. The baseline PF utility and all patients within the PF state in the model were assumed to have a utility of 0.81 based on a previous cost-effectiveness analysis (CEA) of therapies for patients with NDMM ineligible for ASCT. ${ }^{14}$ Disease progression utility of 0.701 was derived from averaged progression utilities of past CEA literature for each treatment. ${ }^{14,27}$ These utilities ranged from 0.27 to 0.73 and were averaged across studies for the base-case estimates (Table 1). These utilities were varied in the PSA.

Disutilities for each treatment arm were found by multiplying the disutility of an $\mathrm{AE}$ by the proportion of patients reporting the $\mathrm{AE}$ and summing them based on the respective $\mathrm{AE}$ for each treatment arm. The health state utility for the 3 treatment arms were subtracted by the disutility of the $\mathrm{AE}$ 
TABLE 2 Summary of Cost-Effectiveness of Lenalidomide and Dexamethasone Double Therapy; Bortezomib, Lenalidomide, and Dexamethasone Triple Therapy; and Daratumumab, Lenalidomide, and Dexamethasone Triple Therapy

\begin{tabular}{l|c|c|c|c|c}
\hline & $\begin{array}{c}\text { Total cost } \\
\mathbf{( \$ )}\end{array}$ & $\begin{array}{c}\text { Incremental } \\
\text { cost } \\
\mathbf{( \$ )}\end{array}$ & $\begin{array}{c}\text { Effectiveness } \\
\text { (PFQALY) }\end{array}$ & $\begin{array}{c}\text { Incremental } \\
\text { effectiveness }\end{array}$ & $\begin{array}{c}\text { ICER }^{\mathrm{a}} \\
\text { (\$/PFQALY) }\end{array}$ \\
\hline Rd standard therapy & 329,867 & - & 1.24 & - & - \\
\hline VRd triple therapy & 385,434 & 55,567 & 1.35 & 0.10 & $530,256^{\mathrm{b}}$ \\
\hline DRd triple therapy & 626,900 & 241,466 & 1.52 & 0.17 & $1,396,318^{\mathrm{b}}$ \\
\hline DRd triple therapy & 626,900 & 297,033 & 1.52 & 0.28 & $1,060,832^{\mathrm{c}}$ \\
\hline
\end{tabular}

aICERs cannot be replicated based on disaggregated results due to rounding.

${ }^{b}$ Compared with the next lowest cost alternative.

'Compared with the lowest cost alternative.

$D R d=$ daratumumab/lenalidomide/dexamethasone; ICER = incremental cost-effectiveness ratio; $P F Q A L Y=$ progression-free quality-adjusted life-year; $R d=$ lenalidomide/dexamethasone;

$V R d=$ bortezomib/lenalidomide/dexamethasone.

experienced by the patients receiving those treatments. Each disutility was weighted based on the percentage of patients who had each $\mathrm{AE}$ in the MAIA and SWOG S0777 trials. The percentage of each $\mathrm{AE}$ in the Rd group was derived from averaging the $\mathrm{AE}$ prevalence in both trials (Table 1). 14,27-32

\section{Results}

\section{COST-EFFECTIVENESS ANALYSIS}

In the base-case analysis, the total treatment costs of Rd standard therapy had the lowest overall cost at $\$ 329,867$, followed by the VRd triple therapy arm at $\$ 385,434$ and DRd triple therapy with the highest overall total cost at $\$ 626,900$ (Table 2). The cost of DRd triple therapy was nearly 90\% higher than Rd standard therapy and almost $62 \%$ more expensive than VRd triple therapy. However, Rd had the least (1.24) PFQALYs, and DRd had the most (1.52) PFQALYs. VRd had 1.35 PFQALYs, which was more than Rd but less than DRd.

Our model demonstrated that $\mathrm{Rd}$ standard therapy is the most cost-effective treatment choice. However, DRd triple therapy and VRd triple therapy were more effective than Rd therapy in reducing progression or death for patients with NDMM ineligible for ASCT. VRd triple therapy was not cost-effective compared with Rd therapy, with an ICER of $\$ 530,256$ per PFQALY. In addition, DRd triple therapy was not cost-effective when compared with VRd triple therapy, with an ICER of $\$ 1,396,318$, nor was it cost-effective compared with $\mathrm{Rd}$ standard therapy (ICER $=\$ 1,060,832$ per PFQALYs). These were not costeffective choices over $\mathrm{Rd}$, when compared against a WTP threshold of $\$ 150,000$ per PFQALY.

\section{SENSITIVITY ANALYSIS}

One-way sensitivity and threshold analysis were conducted on all costs, utilities, and disutilities included in our model, in order to determine which parameters were the most sensitive in our base-case model (Figure 2). When comparing DRd to VRd treatment choices, the overall drug cost per cycle for DRd, the overall drug cost per cycle of VRd, and the utility of PFS were the most sensitive variables, and they accounted for more than $83 \%$ of the variability of the cost-effectiveness model. Reducing the cost per cycle of DRd, increasing the cost per cycle of VRd, and increasing the utility of PFS decreased the ICER of DRd. We found that the cost of DRd triple therapy was the most sensitive in the model, accounting for $41 \%$ of the variability of the cost-effectiveness result, followed by the cost of VRd triple therapy, which accounted for $29 \%$.

When the overall cost per cycle of daratumumab triple therapy was decreased by $20 \%$ from the base-case cost of $\$ 22,352$ to $\$ 17,882$, there was approximately a $55 \%$ decrease in its ICER, from $\$ 1,396,318$ per PFQALY to $\$ 767,619$ per PFQALY, but this is still not cost-effective at a $\$ 150,000$ WTP threshold. In order for DRd to be cost-effective at a WTP threshold of $\$ 150,000$, the overall cost per cycle of daratumumab triple therapy would need to be discounted to $\$ 11,851$, a 53\% total discount from the base-case cost of $\$ 22,352$ compared with $\mathrm{Rd}$ alone. In contrast, VRd triple therapy would be cost-effective compared with $\mathrm{Rd}$ alone, at a WTP of $\$ 150,000$ when the overall cost of therapy is reduced to $\$ 15,373$, an $11 \%$ discount from basecase cost.

We also performed a PSA (Figure 3). The acceptability curve from this analysis confirmed our findings that neither VRd nor DRd triple therapies were cost-effective options compared with Rd. At a WTP of $\$ 150,000$, only $\mathrm{Rd}$ was cost-effective for the majority, $65 \%$ of all iterations. Although, as WTP thresholds increased during the sensitivity analysis, the probability that both DRd and VRd triple therapy became more cost-effective relative to Rd increased, VRd was not cost-effective compared with Rd until a WTP threshold of $\$ 550,000$ was reached. At this increased threshold, VRd became more cost-effective than $\mathrm{Rd}$ for $40 \%$ 


\section{FIGURE 2 Tornado Diagrams for 1-Way Sensitivity Analysis}

\section{A. ICER (\$/PFQALY) - DRd vs Rd}

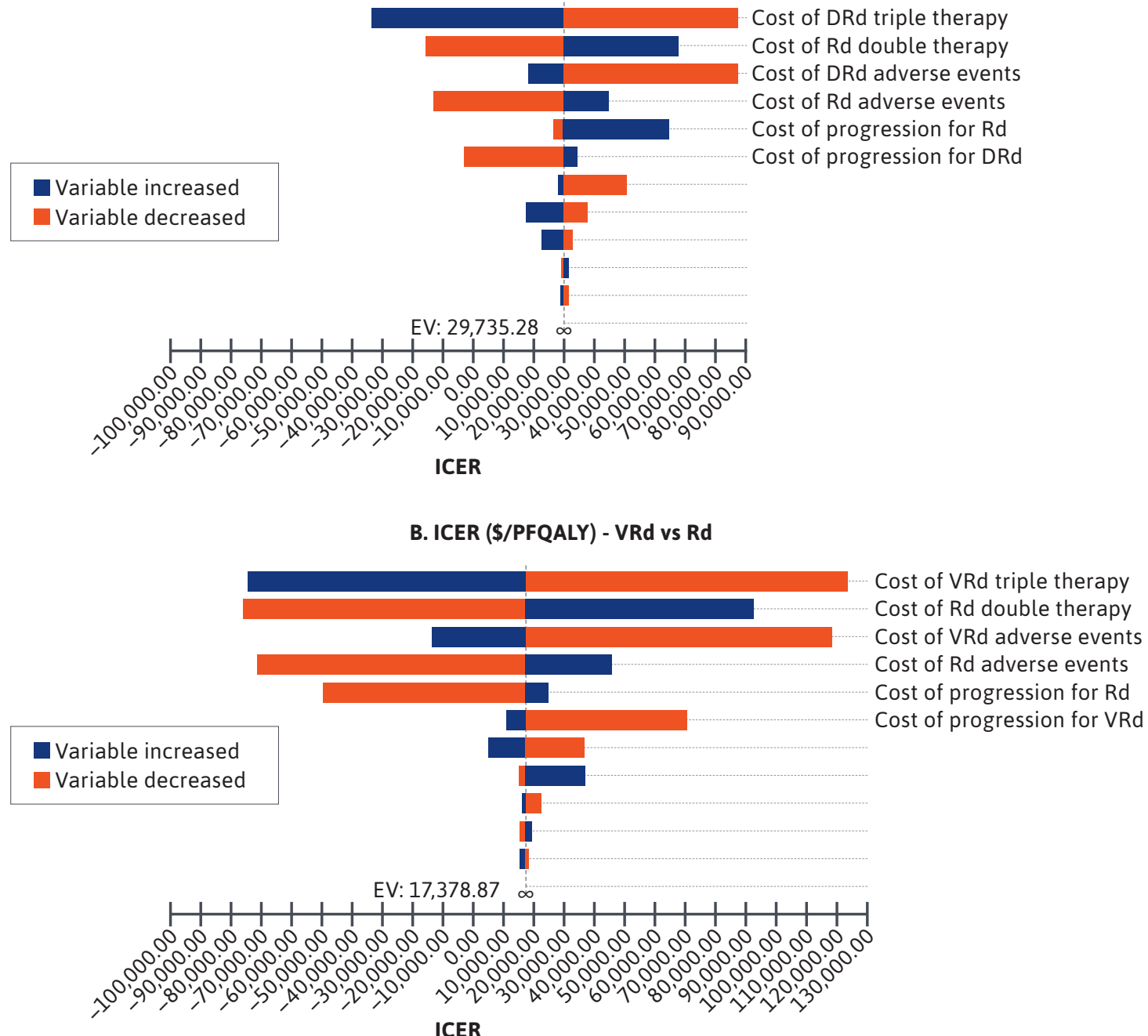

continued on next page

of iterations. DRd was not cost-effective compared with Rd until a WTP threshold of $\$ 750,000$ was reached. At this threshold, DRd became more cost-effective than Rd for $25 \%$ of iterations, while VRd, was still more cost-effective than DRd. There were no reasonable WTP thresholds, up to $\$ 800,00$, where DRd became more cost-effective than VRd.

In our PFLY analysis, the total treatment costs for the 3 groups remained the same (Supplementary Table 3, available in online article). The PFLY saved for DRd triple therapy was longest (2.03 years), whereas for Rd standard therapy PFLY saved was shortest (1.62 years). The overall ICERs for DRd and VRd decreased to $\$ 963,815$ per PFLY and $\$ 360,673$ per PFLY, respectively, but remained above the $\$ 150,000$ WTP threshold.

\section{Discussion}

Our study is the first to date to compare 3 clinically relevant treatments for patients with NDMM ineligible for ASCT, showing that neither DRd nor VRd is cost-effective compared with standard therapy, Rd, and that VRd is more cost-effective than DRd. Rd is a viable treatment regimen based on National Comprehensive Cancer Network guidelines, and it 


\section{FIGURE 2 Tornado Diagrams for 1-Way Sensitivity Analysis (continued)}

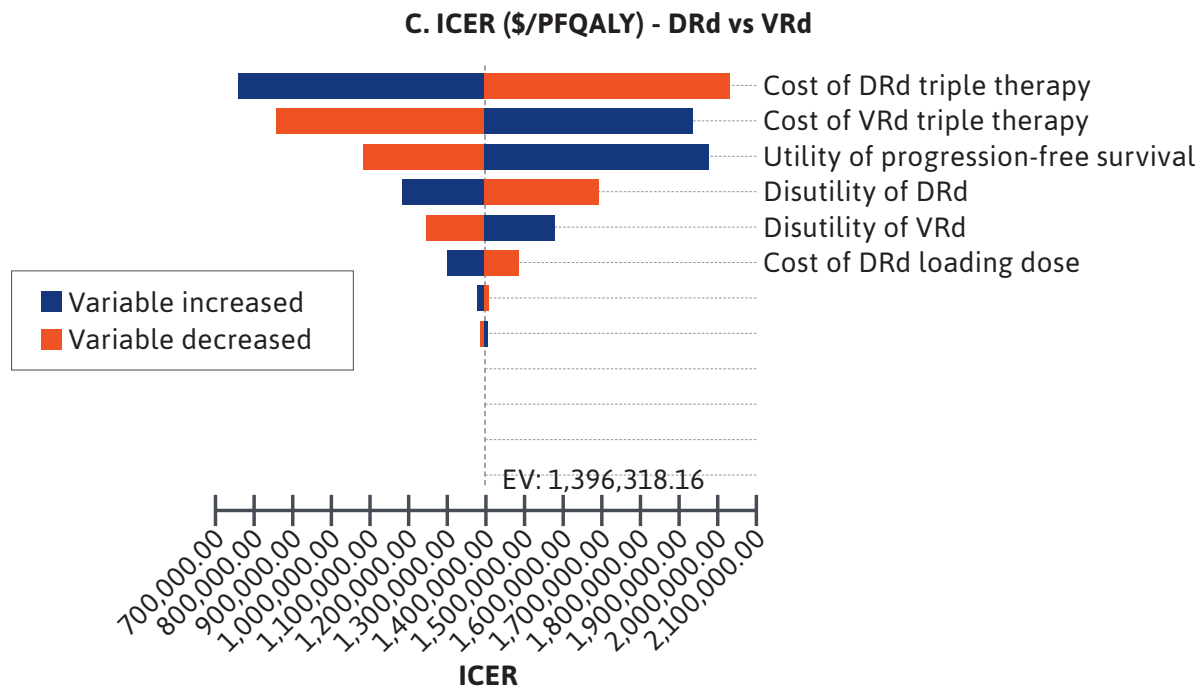

$D R d=$ daratumumab/lenalidomide/dexamethasone; $E V=$ expected value; ICER = incremental cost-effectiveness ratio; PFQALY=progression-free quality-adjusted life-year; $R d=$ lenalidomide/dexamethasone; $V R d=$ bortezomib/lenalidomide/dexamethasone.

is the therapy shown to be the most cost-effective choice. ${ }^{9}$ The high-cost of daratumumab and bortezomib had the most contribution to the lack of cost-effectiveness compared with our other treatment alternatives. ${ }^{33}$

Our results were based on PFQALY rather than QALY, because of the availability of only PFS data for these drugs. Current WTP estimates are based on OS rather than PFS. Therefore, once OS data becomes available across all studied treatments, our model can be restructured to calculate QALYs and allow for comparison with WTP thresholds commonly cited across diseases.

Currently, there is no accepted recommendation for WTP estimates for PF CEA outcomes. However, for nonsmall-cell lung cancer, 1 study showed that access granted to drugs with PFS benefit between 3 and 3.5 months were robustly beneficial across all model parameters, whereas access for drugs with any PFS benefit was usually not beneficial. ${ }^{34}$ Therefore, given that the average PFS length is 4.9 months and 14.9 months in nonsmall-cell lung cancer and multiple myeloma, respectively, we would expect the CEA relative comparison to remain largely unchanged due to the PFS benefit being 3.4 months and 1.3 months for DRd and VRd, respectively. ${ }^{35,36}$ Nevertheless, we recommend conducting an additional CEA when OS data becomes available.
In our analysis, we found an ICER for DRd vs VRd of $\$ 1,396,318$ per PFQALY and vs Rd of $\$ 530,256$ per PFQALY. These results are similar to previous CEA findings that looked at DRd vs Rd in patients with relapsed/refractory multiple myeloma (RRMM). Patients who progressed to RRMM were similar to patients with NDMM ineligible for ASCT because the traditional regimen for both is $\mathrm{Rd}$. In RRMM, the ICER was $\$ 1,369,062$ per QALY for DRd vs Rd. In addition, DRd was found not to be cost-effective under any discount level. ${ }^{14}$ Another CEA looking at DRd vs Rd for second-line therapy in RRMM found an ICER of $\$ 187,728$ per QALY. ${ }^{37}$ DRd as second-line therapy had a lower ICER due to spending less time in the PF health state, therefore, spending less time on treatment. However, even when DRd was prescribed as second-line therapy for RRMM, the ICER did not meet the $\$ 150,000$ WTP threshold.

In order to elucidate the effects of the high cost of drugs, 1-way sensitivity analyses are recommended to provide additional cost-effectiveness information. The cost of DRd triple therapy followed by the cost of VRd triple therapy had the most impact on the overall ICER when comparing DRd with VRd (Figure 2). This is understandable considering that the cost of 1 cycle of DRd and VRd after loading is $\$ 22,352.68$ and $\$ 17,243.13$, respectively (Table 1). In addition, the $3.5 \mathrm{mg}$ vial size for bortezomib is higher than the average dose 


\section{FIGURE 3 Cost-Effective Acceptability Curve}

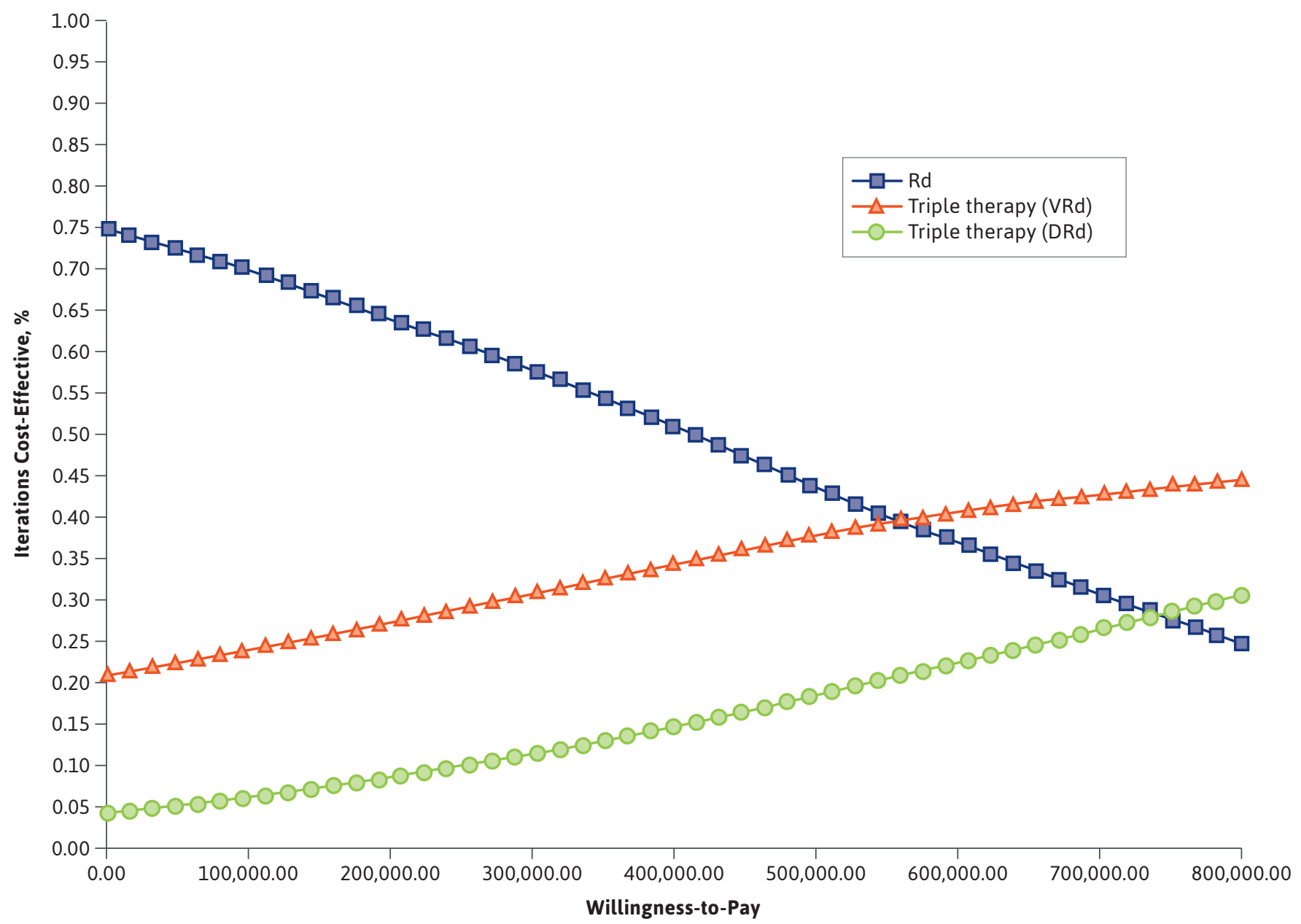

$D R d=$ daratumumab/lenalidomide/dexamethasone; ICER =incremental cost-effectiveness ratio; PFQALY=progression-free quality-adjusted life-year;

$R d=$ lenalidomide/dexamethasone; $V R d=$ bortezomib/lenalidomide/dexamethasone

prescribed, leading to $36.9 \%$ of the dose being wasted for a person with a body surface area of $1.7 \mathrm{~m}^{2} .^{38}$ This amount of wastage was included in our model and was a factor leading to the high ICER for VRd.

Our acceptability curve (Figure 3) was generated by varying drug costs, costs of $\mathrm{AE}$ management, costs of progression, costs of supportive care, utilities of health states, and AE disutilities. Previous acceptability curves for DRd vs Rd in RRMM showed that after the WTP increased to $\$ 1,500,000$ per QALY, DRd had a greater than $50 \%$ probability of being cost-effective compared with the Rd regimen. ${ }^{14}$ This is similar to our findings that DRd and VRd were not cost-effective compared with Rd until a WTP threshold of $\$ 750,000$ per PFQALY and $\$ 550,000$ per PFQALY was reached (Figure 3). Both analyses confirm that the probability of DRd or VRd being cost-effective compared with Rd at a WTP threshold of $\$ 150,000$ per PFQALY was very low.

This CEA analysis can play a role in the decision-making process of clinicians when determining the most costeffective choice for patients with NDMM ineligible for ASCT. However, formulary considerations and channels of distribution for each product also heavily influence treatment choices. Although the addition of daratumumab or bortezomib to Rd may be recommended by the National Comprehensive Cancer Network for "non-frail" patients with NDMM ineligible for ASCT, the results of our CEA 
do not support the addition of daratumumab or bortezomib. ${ }^{9}$ Therefore, clinicians need to strongly consider the benefits vs risks and costs of adding these agents for patients with NDMM ineligible for ASCT.

\section{LIMITATIONS}

This study has some limitations to consider. Given that our model was developed using the MAIA and SWOG S0777 trials, our results are generalizable to patients with similar characteristics as those enrolled in the trials. Also, only the costs for managing grade 3 and 4 AEs, defined as severe/life threatening adverse events that would require hospitalization as well as treatment intervention to resolve, were included. ${ }^{39}$ We chose to exclude the cost of grade 1 and $2 \mathrm{AEs}$ because they were unlikely to contribute significantly to the model due to the low severity. Systematic reviews of immune checkpoint inhibitors, which are a main treatment for many oncologic diseases, show that grade 1-2 AEs are commonly excluded in CEAs. ${ }^{40}$

Without data for the OS curves in the MAIA trial, we did not have the data to distinguish between progression and death health states, which limited our analysis. In addition, each patient incurred the same average progression cost when they entered the progressed or dead health state. The cost of progression included a hospitalization cost due to exacerbation of multiple myeloma symptoms. Considering the multiple therapy options available after progression, we assumed that the choice of treatment after progression was the same between groups.

In addition, our WTP thresholds were based on QALYs, not PFQALYs, for which there are no estimates for comparisons. Therefore, this study should be followed up with an additional analysis once the OS data are available.
Overall, the inclusion of daratumumab or bortezomib with Rd therapy increased the PFS of patients with NDMM ineligible for ASCT. However, the costs of adding these therapies proved to be not cost-effective compared with using Rd alone. Our analysis provides important considerations when choosing value-based treatments for this patient population.

\section{Conclusions}

Despite the limitations, our study was the first to compare daratumumab and bortezomib to current standard of care Rd. The cost-effectiveness for these 2 drugs is mainly inhibited by the high monthly costs of the treatments. Without significant discounts or rebates, $\mathrm{Rd}$ is the more cost-effective option for this patient population. However, because of the promising clinical efficacy of both treatments shown in the MAIA and SWOG S0777 trials, CEAs of these 2 therapies are necessary, since they may have broader clinical uses in other disease states.

Because the AWP of daratumumab and bortezomib may change in the near future due to market competition and possible FDA approval of additional indications, our study could be used to help support the formulary decision making of a health plan when determining the health value of these triple therapies. Our study is the first to compare 3 viable treatment options for patients with NDMM who are ineligible for ASCT, and the added information our study offers can guide health care professionals in choice of treatment regimens.

\section{DISCLOSURES}

No funding was received for this study At the time of this study, Narsipur was a UCSF-Actelion Clinical Research and Medical Communications Fellow, unrelated to this study. The other authors have nothing to disclose.

\section{REFERENCES}

1. Rajkumar SV. MGUS and smoldering multiple myeloma: update on pathogenesis, natural history, and management. Hematol Am Soc Hematol Educ Program. 2005:340-45. doi:10.1182/ asheducation-2005.1.340

2. Zweegman S, Palumbo A, Bringhen S, Sonneveld P. Age and aging in blood disorders: multiple myeloma. Haematologica. 2014;99(7):1133-37. doi:10.3324/

haematol.2014.110296

3. American Cancer Society. Key statistics for multiple myeloma. 2020. Updated January 12, 2021. Accessed October 4, 2020. https://www.cancer.org/cancer/ multiple-myeloma/about/key-statistics. $\underline{\text { html }}$

4. Maiese EM, Evans KA, Chu B-C, Irwin DE. Temporal trends in survival and healthcare costs in patients with multiple myeloma in the United States. Am Health Drug Benefits. 2018;11(1):39-46.

5. National Cancer Institute, SEER. Cancer stat facts: myeloma. Accessed May 7, 2020 https://seer.cancer.gov/statfacts/html/ mulmy.html

6. Attal M, Harousseau J-L, Facon T, et al. Single versus double autologous stem-cell transplantation for multiple myeloma. N Engl J Med. 2003;349(26):2495-502. doi:10.1056/NEJMoa032290

7. Child JA, Morgan GJ, Davies FE, et al. High-dose chemotherapy with hematopoietic stem-cell rescue for multiple myeloma. N Engl J Med. 2003;348(19): 1875-83. doi:10.1056/NEJMoa022340

8. Atrash S, Bhutani M, Paul B, Voorhees PM, Usmani SZ. Management of newly diagnosed transplant ineligible multiple myeloma. Leuk Lymphoma. 2020;61(11):2549-60. doi:10.1080/10428194. 2020.1786558 
9. National Comprehensive Cancer Network. NCCN clinical practice guidelines in oncology - multiple myeloma. Version 1.2022. Accessed August 23, 2020. https://www.nccn.org/guidelines/ guidelines-detail? category $=1 \& \mathrm{id}=1445$

10. Richardson PG, Weller E, Lonial S, et al. Lenalidomide, bortezomib, and dexamethasone combination therapy in patients with newly diagnosed multiple myeloma. Blood. 2010;116(5):679-86. doi:10.1182/blood-2010-02-268862

11. Orlowski RZ, Stinchcombe TE, Mitchell BS, et al. Phase I trial of the proteasome inhibitor ps-341 in patients with refractory hematologic malignancies. J Clin Oncol. 2002;20(22):4420-27. doi:10.1200/JCO.2002.01.133

12. Mateos M-V, Dimopoulos MA, Cavo M, et al. Daratumumab plus bortezomib, melphalan, and prednisone for untreated myeloma. N Engl J Med. 2018;378(6):518-28. doi: 10.1056/NEJMoa1714678

13. Facon T, Kumar S, Plesner T, et al. Daratumumab plus lenalidomide and dexamethasone for untreated myeloma. N Engl J Med. 2019;380(22):2104-15. doi:10.1056/NEJMoa1817249

14. Zhang T-T, Wang S, Wan N, Zhang L, Zhang Z, Jiang J. Cost-effectiveness of daratumumab-based triplet therapies in patients with relapsed or refractory multiple myeloma. Clin Ther. 2018;40(7):1122-39. doi:10.1016/j. clinthera.2018.05.012

15. Durie BGM, Hoering A, Abidi MH, et al. Bortezomib with lenalidomide and dexamethasone versus lenalidomide and dexamethasone alone in patients with newly diagnosed myeloma without intent for immediate autologous stem-cell transplant (SWOG S0777): a randomised, open-label, phase 3 trial. Lancet. 2017;389(10068):519-27. doi:10.1016/ S0140-6736(16)31594-X

16. Darzalex. Prescribing information. Janssen Biotech; 2021. Accessed July 30, 2021. http://www.janssenlabels.com/ package-insert/product-monograph/prescribing-information/DARZALEX-pi.pdf
17. Velcade. Prescribing information. Millennium Pharmaceuticals; 2014. Accessed December 7, 2020. https:// www.accessdata.fda.gov/drugsatfda docs/label/2014/021602s040lbl.pdf

18. Pelligra CG, Parikh K, Guo S, et al. Cost-effectiveness of pomalidomide, carfilzomib, and daratumumab for the treatment of patients with heavily pretreated relapsed-refractory multiple myeloma in the United States. Clin Ther. 2017;39(10):1986-2005.e5. doi: 10.1016/j. clinthera.2017.08.010.

19. Neumann PJ, Cohen JT, Weinstein MC. Updating cost-effectiveness-the curious resilience of the $\$ 50,000-$ per-QALY threshold. N Engl J Med. 2014;371(9):79697. doi:10.1056/NEJMp1405158

20. Hoyle MW, Henley W. Improved curve fits to summary survival data: application to economic evaluation of health technologies. BMC Med Res Methodol. 2011;11(1):139. doi:10.1186/1471-2288-11-139

21. Congressional Budget Office. Prescription drug pricing in the private sector. January 2007. Accessed October 13, 2021. https://www.cbo.gov/sites/ default/files/110th-congress-2007-2008/ reports/01-03-prescriptiondrug.pdf

22. Sanders GD, Neumann PJ, Basu A, et al. Recommendations for conduct, methodological practices, and reporting of cost-effectiveness analyses: Second Panel on Cost-Effectiveness in Health and Medicine. JAMA. 2016;316(10):1093-103. doi:10.1001/jama.2016.12195

23. UPMC. Billing terms and definitions. Accessed July 29, 2021. https://www. upmc.com/patients-visitors/paying-bill/ glossary

24. Healthcare Cost and Utilization Project. SID Overview. Agency for Healthcare Research and Quality. Accessed July 29, 2021. https://www. hcup-us.ahrq.gov/sidoverview.jsp

25. U.S. Bureau of Labor Statistics. How to use the Consumer Price Index for escalation. Revised November 25, 2020. Accessed July 29, 2021. https://www.bls. gov/cpi/factsheets/escalation.htm
26. Cömert M, Güneş AE, Şahin F, Saydam G. Quality of life and supportive care in multiple myeloma. Turk J Hematol. 2013;30(3):234-46. doi:10.4274/ Tjh.2012.0192

27. Kouroukis T, White D, Kruse M, Lawrence D, Trambitas C, Cheung MC. Cost-utility of bortezomib in induction treatment prior to autologous stem-cell transplantation (ASCT) in previously untreated multiple myeloma patients in Canada. Blood. 2013;122(21):1735-35. doi:10.1182/blood.V122.21.1735.1735

28. Ossa DF, Briggs A, McIntosh E, Cowell W, Littlewood T, Sculpher M. Recombinant erythropoietin for chemotherapy-related anaemia: economic value and health-related quality-of-life assessment using direct utility elicitation and discrete choice experiment methods. PharmacoEconomics. 2007;25(3):223-37. doi:10.2165/00019053-200725030-00005

29. Büyükkaramikli NC, de Groot $S$, Fayter D, et al. Pomalidomide with dexamethasone for treating relapsed and refractory multiple myeloma previously treated with lenalidomide and bortezomib: an evidence review group perspective of an NICE single technology appraisal. Pharmacoeconomics. 2018;36(2):145-59. doi:10.1007/ s40273-017-0581-6

30. Doyle S, Lloyd A, Walker M. Health state utility scores in advanced nonsmall cell lung cancer. Lung Cancer Amst Neth. 2008;62(3):374-80. doi:10.1016/j. lungcan.2008.03.019

31. Franic DM, Pathak DS, Gafni A. Are health states "timeless"? A case study of an acute condition: postchemotherapy nausea and vomiting. J Eval Clin Pract. 2003;9(1):69-82. doi:10.1046/j.1365-2753.2003.00381.x

32. Beusterien KM, Szabo SM, Kotapati S, et al. Societal preference values for advanced melanoma health states in the United Kingdom and Australia. Br J Cancer. 2009;101(3):387-89. doi:10.1038/ sj.bjc. 6605187 
33. Institute for Clinical and Economic Review. Value assessment framework. Accessed January 23, 2021. https://icer. org/our-approach/methods-process/ value-assessment-framework/

34. Lakdawalla DN, Chou JW, Linthicum MT, MacEwan JP, Zhang J, Goldman DP. Evaluating expected costs and benefits of granting access to new treatments on the basis of progressionfree survival in non-small-cell lung cancer. JAMA Oncol. 2015;1(2):196-202. doi:10.1001/jamaoncol.2015.0203

35. Hayashi H, Okamoto I, Morita S, Taguri M, Nakagawa K. Postprogression survival for first-line chemotherapy of patients with advanced non-small-cell lung cancer. Ann Oncol Off J Eur Soc Med Oncol. 2012;23(6):1537-41. doi:10.1093/ annonc/mdr487
36. Verelst SGR, Blommestein HM, De Groot S, et al. Long-term outcomes in patients with multiple myeloma: a retrospective analysis of the Dutch Population-based HAematological Registry for Observational Studies (PHAROS). HemaSphere. 2018;2(4):e45. doi:10.1097/HS9.0000000000000045

37. Carlson JJ, Guzauskas GF, Chapman RH, et al. Cost-effectiveness of drugs to treat relapsed/refractory multiple myeloma in the United States. J Manag Care Spec Pharm. 2018;24(1):29-38. doi:10.18553/ jmcp.2018.24.1.29
38. Clark L, Castro AP, Fortes AF, et al. Ideal vial size for bortezomib: real-world data on waste and cost reduction in treatment of multiple myeloma in Brazil. Value Health. 2011;14(5):S82-S84. doi:10.1016/j. jval.2011.05.013

39. Russell JS, Colevas AD. Adverse event monitoring in oncology clinical trials. Clin Investig. 2013;3(12):1157-65. doi:10.4155/ cli.13.111

40. Verma V, Sprave T, Haque W, et al. A systematic review of the cost and cost-effectiveness studies of immune checkpoint inhibitors. J Immunother Cancer. 2018;6(1):128. doi:10.1186/ s40425-018-0442-7 